\title{
Bei Schwindelpatienten ist oft schon die Anamnese wegweisend
}

\author{
Bei Patienten, die über Schwindel klagen, kann neben einer ausführ- \\ lichen Anamnese das Führen eines Schwindeltagebuchs sinnvoll sein, \\ so die Erfahrung von Dr. Elfi Seeger-Schellerhoff, Fachärztin für Hals- \\ Nasen-Ohren-Heilkunde aus Porta Westfalica.
}

\begin{abstract}
? Welche Patienten sind typischerweise von Schwindel betroffen?

Seeger-Schellerhoff: Den typischen Schwindelpatienten gibt es eigentlich nicht. Es können alle Altersgruppen betroffen sein. Bei älteren und/oder multimorbiden Patienten treten Schwindelanfälle jedoch vermehrt auf. Diese Patienten leiden unter ihren Beschwerden zum Teil ganz erheblich, weil sie sich in ihrem Alltag beeinträchtigt fühlen und Angst haben zu stürzen und sich dabei ernsthaft zu verletzen.
\end{abstract}

?Hat die Zahl der Patienten mit Schwindel nach Ihrer Beobachtung im Laufe der Jahre zugenommen?

Seeger-Schellerhoff: Ja, das kann man schon sagen. Die Entwicklung ist deutlich progredient. Das liegt sicher auch daran, dass die Patienten heute im Schnitt deutlich älter sind. Die Patienten achten heute aber auch mehr auf ihr Befinden und nehmen solche Störungen wie etwa Schwindel sehr viel stärker wahr als noch vor einigen Jahren. Ähnliches sehen wir ja auch beim Tinnitus. Früher hatte man halt Ohrgeräusche und hat das nicht weiter artikuliert. Heute sind die Menschen besser aufgeklärt. Natürlich spielt auch die Einnahme von Medikamenten eine Rolle.

?Worauf sollte man bei der Diagnose besonders achten?

Seeger-Schellerhoff: Ganz wichtig sind eine gute Anamnese und die Schilderung der Beschwerden durch den Patienten. Dafür sollte man sich Zeit nehmen. Die Patienten sollen mit ihren eigenen Worten schildern, wie sie den Schwindel erleben. In unserer Praxis lassen wir die Patienten im Wartezimmer zusätzlich einen Schwindel-
Anamnesebogen ausfüllen, den wir dann anschließend gemeinsam besprechen. $\mathrm{Na}$ türlich muss jeder Patient auf allen Ebenen, also auch neurologisch und internistisch, abgeklärt werden. Außerdem sehe ich mir bei jedem Patienten die Medikation an.

?Worüber klagen Patienten mit unspezifischem Schwindel typischerweise?

Seeger-Schellerhoff: Manche können ihre Beschwerden gar nicht so genau beschreiben. Andere berichten, dass sie sich beim Stehen oder Gehen sehr unsicher fühlen, dass innen schwarz vor Augen wird, dass der Kopf "leer" ist und dass sie Angst haben, deshalb zu stürzen.

?Worin unterscheiden sich jüngere und ältere Patienten mit Schwindel?

Seeger-Schellerhoff: Bei den jüngeren Patienten handelt es sich meist um vestibuläre Schwindelattacken. Das ist bei den älteren Patienten eher selten. Ältere Patienten sind meist in der Diagnostik völlig unauffällig. Diese Patienten stellen wir dann auch gerne auf Vertigoheel ${ }^{\circledast}$ ein.

?Welche Erfahrungen haben Sie mit dem natürlichen Komplexmittel?

Seeger-Schellerhoff: Bei uns bekommen die Patienten mit diffusem Schwindel, die auf Vertigoheel ${ }^{\oplus}$ eingestellt werden, in der ersten Woche $6 \times 1$ Tablette täglich, zeitversetzt zu ihrer normalen Medikation, über den Tag verteilt. Nach einer Woche wird die Dosis dann auf 3 x 1 Tablette täglich reduziert. Die Patienten werden grundsätzlich nach vier bis sechs Wochen wieder einbestellt, um den Therapieerfolg zu beurteilen. Sehr wichtig ist es, dass die Patienten immer auch häusliche praktische Gleichgewichts-

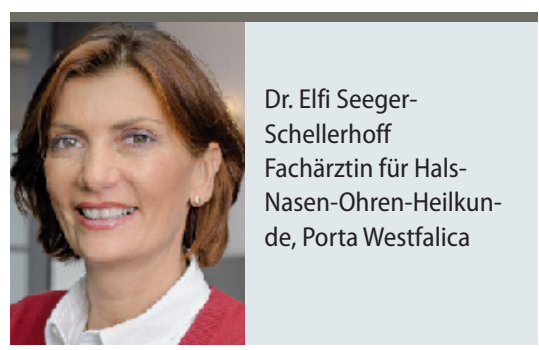

übungen machen, die sie problemlos in den Tagesablauf integrieren können. Zum Beispiel: Zähneputzen auf einem Bein stehend, auf einem Stuhl sitzend Drehübungen mit dem Kopf machen, Blickfixationsübungen und Laufübungen. Wir haben damit sehr gute Erfahrungen gemacht, zumal die Patienten im Rahmen ihrer Möglichkeiten auch selbst aktiv sein wollen.

?Was können die Patienten darüber hinaus noch tun?

Seeger-Schellerhoff: Unsere Patienten führen auch ein Schwindeltagebuch, in dem sie notieren, was den Schwindel ausgelöst hat, wie lange er gedauert hat, was für ein Schwindel das war und ob sie gestürzt sind. Wenn möglich sollten sie auch den Blutdruck messen. Aus einem solchen Tagebuch kann man den Verlauf des Schwindels auch nach zwei oder drei Wochen besser nachvollziehen und unter Umständen Muster erkennen, die dem Patienten vielleicht gar nicht bewusst sind.

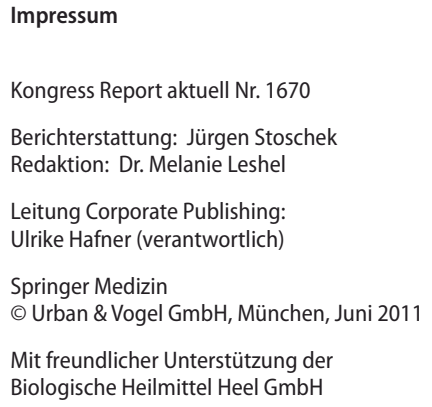

